\title{
Probability Measures on Semigroups
}

Convolution Products, Random Walks, and Random Matrices 


\section{THE UNIVERSITY SERIES IN MATHEMATICS}

Series Editors: Sylvain E. Cappell, New York University Joseph J. Kohn, Princeton University

Recent volumes in the series:

THE CLASSIFICATION OF FINITE SIMPLE GROUPS

Daniel Gorenstein

VOLUME 1: GROUPS OF NONCHARACTERISTIC 2 TYPE

COMPLEX ANALYSIS AND GEOMETRY

Edited by Vincenzo Ancona and Alessandro Silva

ELLIPTIC DIFFERENTIAL EQUATIONS AND OBSTACLE PROBLEMS

Giovanni Maria Troianiello

FINITE SIMPLE GROUPS: An Introduction to Their Classification

Daniel Gorenstein

\section{AN INTRODUCTION TO ALGEBRAIC NUMBER THEORY}

Takashi Ono

\section{MATRIX THEORY: A Second Course}

James M. Ortega

PROBABILITY MEASURES ON SEMIGROUPS: Convolution Products, Random Walks, and Random Matrices

Göran Högnäs and Arunava Mukherjea
A SCRAPBOOK OF COMPLEX CURVE THEORY
C. Herbert Clemens

\section{TOPICS IN NUMBER THEORY}

J. S. Chahal

VARIATIONS ON A THEME OF EULER

Quadratic Forms, Elliptic Curves, and Hopf Maps

Takashi Ono

A Continuation Order Plan is available for this series. A continuation order will bring delivery of each new volume immediately upon publication. Volumes are billed only upon actual shipment.

For further information please contact the publisher. 


\section{Probability Measures on Semigroups}

Convolution Products, Random Walks, and Random Matrices

Göran Högnäs Ảbo Akademi University Abo, Finland
Arunava Mukherjea

University of South Florida

Tampa, Florida 


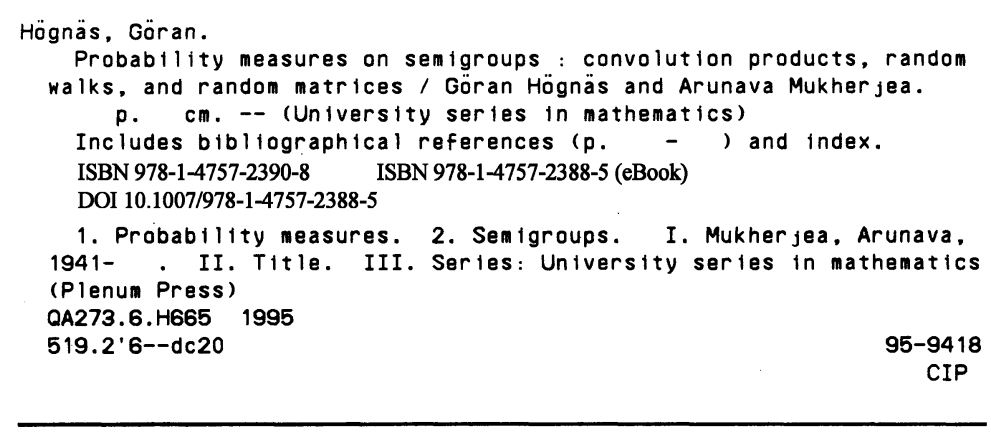

C1995 Springer Science+Business Media New York

Originally published by Plenum Press, New York in 1995

Softcover reprint of the hardcover 1st edition 1995

\section{1}

All rights reserved

No part of this book may be reproduced, stored in a retrieval system, or transmitted in any form or by any means, electronic, mechanical, photocopying, microfilming, recording, or otherwise, without written permission from the Publisher 


\section{Preface}

A Scientific American article on chaos, see Crutchfield et al. (1986), illustrates a very persuasive example of recurrence. A painting of Henri Poincaré, or rather a digitized version of it, is stretched and cut to produce a mildly distorted image of Poincaré. The same procedure is applied to the distorted image and the process is repeated over and over again on the successively more and more blurred images. After a dozen repetitions nothing seems to be left of the original portrait. Miraculously, structured images appear briefly as we continue to apply the distortion procedure to successive images. After 241 iterations the original picture reappears, unchanged!

Apparently the pixels of the Poincare portrait were moving about in accordance with a strictly deterministic rule. More importantly, the set of all pixels, the whole portrait, was transformed by the distortion mechanism. In this example the transformation seems to have been a reversible one since the original was faithfully recreated.

It is not very farfetched to introduce a certain amount of randomness and irreversibility in the above example. Think of a random miscoloring of some pixels or of inadvertently giving a pixel the color of its neighbor.

The methods in this book are geared towards being applicable to the asymptotics of such transformation processes. The transformations form a semigroup in a natural way; we want to investigate the long-term behavior of random elements of this semigroup.

To be more specific, let us consider a sequence of independent and identically distributed random variables $X_{0}, X_{1}, X_{2}, \ldots$ taking values in a set of affine maps from $R^{d}$ into $R^{d}$, that is, maps of the form $f(x)=A x+B$, where $B$ and $x$ are $d \times 1$ column vectors and $A$ is a $d \times d$ real matrix. Since $f$ can be identified with the $(d+1) \times(d+1)$ matrix $\left(\begin{array}{cc}A & B \\ 0 & 1\end{array}\right)$, the random variables 
$X_{i}$ s can also be regarded as $(d+1) \times(d+1)$ random matrices; thus, $\mu$, the distribution of $X_{i}$, is a probability measure on the set of $(d+1) \times(d+1)$ matrices of the form $\left(\begin{array}{cc}A & B \\ 0 & 1\end{array}\right)$. Let $S$ be the closed (with usual topology) multiplicative semigroup generated by the support of $\mu$. Then the study of the random walks $Y_{n}, Y_{n}=X_{0} X_{1} \ldots X_{n}$ with values in $S$ and distribution $\mu^{n}$ (the $n$th convolution power of $\mu$ ), and the set of recurrent states of $\left(Y_{n}\right)$ become relevant in the context of the so-called iterated function systems introduced by Barnsley and his colleagues [see Barnsley (1988)].

Let us briefly discuss another example.

Suppose that we are monitoring a random system with two states denoted 0 and 1. Let

and

\section{0}

be observed time series of the successive states of the system. The observations seem rather like a record of independent coin tosses, with 0 for heads and 1 for tails, say. Viewed as a Markov chain on the two-state state space $X=\{0,1\}$ our process would have the transition probability matrix

$$
P=\left(\begin{array}{cc}
1 / 2 & 1 / 2 \\
1 / 2 & 1 / 2
\end{array}\right)
$$

Let us assume, however, that the above time series are concurrent. Then another interpretation imposes itself: the state space is subjected to a succession of random transformations. (The first two transformations are transpositions, 0 and 1 just trade places. At the third and fourth steps the identity map is at work. A sequence of transpositions and identities then follows, but at step 19 everything is mapped onto the state 1 . From then on the two paths are identical.) The transformations are the four possible mappings of $X$ into itself, the identity $\iota$, the transposition $\tau$, and the two constant mappings 0 and 1 . The transition matrix $P$ is then a convex combination of matrices representing those transformations:

$$
P=a\left(\begin{array}{ll}
1 & 0 \\
0 & 1
\end{array}\right)+b\left(\begin{array}{ll}
0 & 1 \\
1 & 0
\end{array}\right)+c\left(\begin{array}{ll}
1 & 0 \\
1 & 0
\end{array}\right)+d\left(\begin{array}{ll}
0 & 1 \\
0 & 1
\end{array}\right),
$$

where $a, b, c, d$ are nonnegative numbers with $a+b+c+d=1$.

Thus, a natural way to analyze our observed time series is to think of them as emanating from an independent, identically distributed sequence of mappings 
of the state space into itself, or, in other words, a random walk on the transformations of $X$.

A Markov chain on a finite state space can always be regarded in this way. Its transition matrix $P$ is a convex combination of $0-1$ matrices representing mappings of the state space $X$ into itself. (If $P$ is doubly stochastic we can write it uniquely as a convex combination of permutation matrices, this is the celebrated Birkhoff theorem.) The corresponding result is true even for a large class of Markov chains on a topological space $X$, see Kifer (1986), Chapter 1 .

To consider an example in the context of particle systems, let $V$ be an arbitrary countably infinite set (with discrete topology), and let $\Gamma$ denote the semigroup of functions $f: V \rightarrow V$ under composition. We can then identify each $f$ in $\Gamma$ with an infinite $0-1$ stochastic matrix $A_{f}$ such that

$$
\left(A_{f}\right)_{i j}=1 \text { if and only if } f(i)=j .
$$

By a configuration $\eta$ of $V$, we mean a nonnegative integer valued function on $V$ such that

$$
\sum_{x \in V} \eta(x)<\infty
$$

The idea is that $\eta(j)$ is the number of particles that occupy the site $j \in V$, and that when we apply the mapping $f: V \rightarrow V$, all these particles move to the site $f(j)$, and the configuration changes to $\eta \cdot A_{f}$, where

$$
\left(\eta \cdot A_{f}\right)(x)=\sum_{y} \eta(y) \cdot \delta_{f(y)}(x) .
$$

The new configuration has at site $x$ all the particles that the map $f$ has sent to the site $x$ from the sites of the original configuration. Thus, to study the random motions of finite systems of particles on $V$, without births or deaths, where each site may be occupied by a finite number of particles, and all particles at a particular site move together, one needs to study the random transformations $F$ (that is, the infinite random stochastic matrices $A_{F}$ ). Instead of studying the different configurations, we study a sequence of independent identically distributed countably infinite stochastic matrices, and among other things, will be interested in gaining some insights in the limiting laws of products of these matrices.

To mention yet another context where probability measures on countable semigroups have been found useful, we mention the paper of Hansel and Perrin (1983), where the authors utilized the structure of an idempotent probability measure on a semigroup in order to have some insights in certain problems in coding theory.

It is also relevant to mention that Ruzsa (1994) utilized his results on weak* convergence of the sequence $\mu_{1} * \mu_{2} * \ldots * \mu_{n}$, where the $\mu_{i} \mathrm{~s}$ are probability measures on a countable semigroup, in proving a generalization of a result in 
number theory due to Davenport and Erdös (1936). This last mentioned result simply says that every multiplicative ideal $A$ of the set $N$ of positive integers has a logarithmic density, that is,

$$
\lambda(A)=\lim _{n \rightarrow \infty} \frac{1}{\log n} \sum_{\substack{a \in A \\ a \leq n}} \frac{1}{a},
$$

exists. Note that for a set $A \subset N$, its logarithmic density $\lambda$ may exist while its asymptotic density $d$, given by

$$
d(A)=\lim _{n \rightarrow \infty} \frac{1}{n} \sum_{\substack{a \in A \\ a \leq n}} 1,
$$

may not exist. [It is well known, however, that $\lambda(A)$ exists whenever $d(A)$ does, and then $\lambda(A)=d(A)$.] Ruzsa's result says the following: if $f$ is a homomorphism from the multiplicative semigroup of integers to a commutative semigroup $H$, then for every $h \in H$, the set $\{n \in N: f(n)=h\}$ has a logarithmic density.

Let us finally mention, before we go to the text proper, that abstract semigroup theory was of crucial importance in developing the methods used in Högnäs and Mukherjea (1980) to study the set of recurrent states of a random walk taking values in $n \times n$ real matrices.

Now to describe the contents of this book, let us say that here we make an attempt to present up-to-date information in the theory of weak convergence of convolution products of probability measures on semigroups (Chapter 2), the theory of random walks with values in semigroups (Chapter 3), and applications of the preceding theories to products of random matrices (Chapter 4). Chapter 1 contains essentials of abstract semigroup theory along with its application to concrete semigroups of matrices. Chapter 1 , while it contains many important results from abstract semigroup theory, is not designed to cover semigroup theory in depth, and as such contains mostly those results and concepts which are needed for an understanding of later chapters.

To restrict the size of the book, we have often been biased towards presenting only those results which are new, surprising, useful, and interesting in the context of semigroups. However, certain results have been presented in groups rather than semigroups, and this has been done whenever the corresponding semigroup situation is not very clear or so far only partially solved. Thus, we have discussed concentration functions (in Chapter 2) only in the context of groups.

A graduate student familiar with material covered in standard courses (in a typical American university) in probability theory, measure theory, group theory, topology, and linear algebra should not have any difficulty in following this book 
on his/her own. A two-semester special topics course on weak convergence and random walks can be based on the material covered in this book.

For ease of reading, let us also mention that the theorems, lemmas, propositions, and corollaries in each chapter of this book are numbered consecutively in the order in which they appear. Thus, Proposition 2.18 follows Corollary 2.17, Lemma 2.20 follows Proposition 2.19, and Theorem 2.23 follows Lemma 2.22.

Finally, let us express our gratitude to many of our colleagues and friends for assisting us in various ways (in the writing of this book) through discussions and actual collaboration in research. We are specially indebted to Herbert Heyer, Karl Hofmann, Imre Ruzsa, Murray Rosenblatt, T. C. Sun, and Nicolas Tserpes.

Göran Högnäs and Arunava Mukherjea

\section{References}

Barnsley, M. F., Fractals Everywhere, Academic Press, Orlando (1988).

Crutchfield, J. P. , J. D. Farmer, N. H. Packard, and R. S. Shaw, "Chaos," Scientific American 255, No. 6, 38-49 (1986).

Hansel, G. and D. Perrin, "Codes and Bernoulli partitions," Math. Systems Theory 16, 133-157 (1983).

Högnäs, G. and A. Mukherjea, "Recurrent random walks and invariant measures on semigroups of $n \times n$ matrices," Math. Zeitschrift 173, 69-94 (1980).

Kifer, Y., Ergodic Theory of Random Transformations, Birkhäuser, Boston-Basel-Stuttgart (1986).

Ruzsa, I. Z., "Logarithmic density and measures on semigroups," (Preprint) (1994). 


\section{Contents}

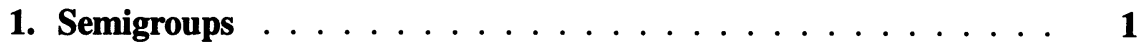

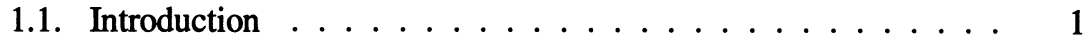

1.2. Homomorphisms, Quotients, and Products . . . . . . . . . 5

1.3. Semigroups with Zero . . . . . . . . . . . . . . . . . 9 9

1.4. Rees-Suschkewitsch Representation Theorem . . . . . . . . . 11

1.5. Topological Semigroups . . . . . . . . . . . . . . . . 22

1.6. Semigroups of Matrices . . . . . . . . . . . . . . 34

1.7. Semigroups of Infinite Dimensional Matrices . . . . . . . 52

1.8. Embedding Semigroups in a Group . . . . . . . . . . . 60

1.9. Notes and Comments . . . . . . . . . . . . . . 62

References ..................... 63

2. Probability Measures on Topological Semigroups _ . . . . . . . 67

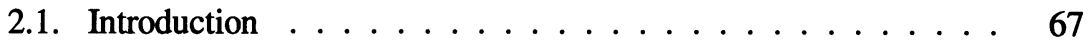

2.2. Invariant and Idempotent Probability Measures . . . . . . . 68

2.3. Weak Convergence of Convolution Products of Probability Measures . . . . . . . . . . . . . . . . . . 87

2.4. Weak Convergence of Convolution Products of Nonidentical Probability Measures . . . . . . . . . . . . . . . . . . 139

2.5. Notes and Comments . . . . . . . . . . . . . . 168

References . . . . . . . . . . . . . . . . . . 169

3. Random Walks on Semigroups $\ldots \ldots \ldots \ldots$. . . . . . . 173

3.1. Introduction . . . . . . . . . . . . . . 173

3.2. Discrete Semigroups . . . . . . . . . . . . . . . . . 183

3.3. Locally Compact Groups . . . . . . . . . . . . . . . . . . 202

3.4. Compact Semigroups . . . . . . . . . . . . . . . . 227 
3.5. Completely Simple Semigroups . . . . . . . . . . . . . 250

3.6. Notes and Comments . . . . . . . . . . . . . . . 256

References ..................... . 260

4. Random Matrices . . . . . . . . . . . . . . . . . . 263

4.1. Introduction . . . . . . . . . . . . . . . . . 263

4.2. Recurrent Random Walks in Nonnegative Matrices . . . . . . 263

4.3. Tightness of Products of I.I.D. Random Matrices: Weak

Convergence . . . . . . . . . . . . . . . . . . . 292

4.4. Invariant Measures for Random Walks in Nonnegative Matrices:

Laws of Large Numbers . . . . . . . . . . . . . . . . . 342

4.5. Asymptotic Behavior of $\left\|X_{n} X_{n-1} \ldots X_{0} u\right\|$ for I.I.D. Random

Nonnegative Matrices . . . . . . . . . . . . . . 365

4.6. Notes and Comments . . . . . . . . . . . . . . . . 377

References . . . . . . . . . . . . . . . . . 379

Index $\ldots \ldots \ldots \ldots \ldots \ldots \ldots \ldots \ldots \ldots \ldots$ 